\title{
888.
}

\section{ON A FORM OF QUARTIC SURFACE WITH TWELVE NODES.}

[From the British Association Report, (1886), pp. 540, 541.]

Using throughout capital letters to denote homogeneous quadric functions of the coordinates $(x, y, z, w)$, we have as a form of quartic surface with eight nodes $\Omega=(* \gamma U, V, W)^{2}=0$; viz. the nodes are here the octad of points, or eight points of intersection of the quadric surfaces $U=0, V=0, W=0$; the equation can, by a linear transformation on the functions $U, V, W$ (that is, by substituting for the original functions $U, V, W$ linear functions of these variables), be reduced to the form $\Omega=U^{2}+V^{2}+W^{2}=0$.

Suppose now that the function $\Omega$ can in a second manner be expressed in the like form $\Omega=P^{2}+Q^{2}+R^{2}$ (where $P, Q, R$ are not linear functions of $U, V, W$ ); that is, suppose that we have identically $U^{2}+V^{2}+W^{2}=P^{2}+Q^{2}+R^{2}$, this gives $U^{2}-P^{2}+V^{2}-Q^{2}+W^{2}-R^{2}=0$; or, writing $U+P, V+Q, W+R=A, B, C$, and $U-P$, $V-Q, W-R=F, G, H$, the identity becomes $A F+B G+C H=0$; and this identity being satisfied, the equation $\Omega=0$ of the quartic surface may be written in the two forms

and

$$
\Omega=\left(A+F^{\top}\right)^{2}+(B+G)^{2}+(C+H)^{2}=0,
$$

$$
\Omega=\left(A-F^{\prime}\right)^{2}+(B-G)^{2}+(C-H)^{2}=0 ;
$$

viz. the quartic surface has the nodes which are the intersections of the three quadric surfaces $A+F=0, B+G=0, C+H=0$, and also the nodes which are the intersections of the three quadric surfaces $A-F=0, B-G=0, C-H=0$. We may of course also write the equation of the surface in the form

$$
\Omega=A^{2}+B^{2}+C^{2}+F^{2}+G^{2}+H^{2}=0 .
$$

C. XIII. 
An easy way of satisfying the identity $A F^{\prime}+B G+C H=0$ is to assume

$$
A, B, C, F, G, H=a y z, b z x, c x y, f x w, g y w, h z w,
$$

where the constants $a, b, c, f, g, h$ satisfy the condition $a f+b g+c h=0$; this being so, the functions $A, B, C, F, G, H$, and consequently the functions $A+F, B+G, C+H$ and $A-F, B-G, C-H$ each of them vanish for the four points $(y=0, z=0, w=0)$, $(z=0, x=0, w=0),(x=0, y=0, w=0),(x=0, y=0, z=0)$, or say the points $(1,0,0,0)$, $(0,1,0,0),(0,0,1,0),(0,0,0,1)$. It hence appears that the quartic surface

$$
\Omega=a^{2} y^{2} z^{2}+b^{2} z^{2} x^{2}+c^{2} x^{2} y^{2}+f^{2} x^{2} w^{2}+g^{2} y^{2} w^{2}+h^{2} z^{2} w^{2}=0
$$

is a quartic surface with twelve nodes; viz. it has as nodes the last-mentioned four points, the remaining four points of intersection of the surfaces

$$
a y z+f x w=0, \quad b z x+g y w=0, \quad c x y+h z w=0,
$$

and the remaining four points of intersection of the surfaces

$$
a y z-f x w=0, \quad b z x-g y w=0, \quad c x y-h z w=0 .
$$

The above is the analytical theory of one of the two forms of quartic surface with twelve nodes recently established by $\mathrm{Dr} \mathrm{K}$. Rohn in a paper in the Berichte ü. d. Verhandlungen der K. Sächsische Gesellschaft zu Leipzig, (1884), pp. 52-60. 